Chair for Management Sciences and Energy Economics University of Duisburg-Essen

EWL Working Paper No. 2

\title{
PORTFOLIO OPTIMIZATION IN ELECTRICITY TRADING WITH LIMITED LIQUIDITY
}

by

Christoph Weber

and

Oliver Woll

July 2007 


\title{
Portfolio optimization in electricity trading with limited liquidity
}

\author{
by Christoph Weber and Oliver Woll
}

\begin{abstract}
In principle, portfolio optimization in electricity markets can make use of the standard mean-variance model going back to Markowitz. Yet a key restriction in most electricity markets is the limited liquidity. Therefore the standard model has to be adapted to cope with limited liquidity. An application of this model shows that the optimal hedging strategy for generation portfolios is strongly dependent on the size of the portfolio considered as well as on the variance-covariancematrix used and the liquidity function assumed.
\end{abstract}

Keywords : optimization; electricity, liquidity; electricity trading; mean-variance-model JEL-Classification : C61, G11, Q40

History: This paper was first submitted 31 July 2007.

\section{PROF. DR. ChRISTOPH WEBER}

Chair for Management Sciences and Energy Economics, University of Duisburg-Essen (Campus Essen)

Universitätsstr. 11, 45117 Essen

++49 - (0)2 01 / 183-2966

www.ewl.wiwi.uni-due.de

christoph.weber@uni-due.de
DIPL.-KFM. OLIVER WOLL

Chair for Management Sciences and Energy Economics, University of Duisburg-Essen (Campus Essen) Universitätsstr. 11, 45117 Essen ++49 - (0)2 01 / 183-3389 www.ewl.wiwi.uni-due.de oliver.woll@uni-due.de

The authors are solely responsible for the contents which do not necessarily represent the opinion of the Chair for Management Sciences and Energy Economics. 


\section{Introduction}

Electricity markets have been liberalised around the globe during the past two decades. This has led utilities to consider their assets not only as technical devices to fulfill obligations of load servicing but rather as assets, which can be used to maximize value for shareholders or/and society. But liberalisation has also led to previously unknown levels of price volatility in the electricity markets, and the companies have had to learn that they have to cope efficiently with these risks. The Californian energy crisis, the Enron collapse, but also less prominent show cases have provided ample evidence that electricity price risks may have disastrous effects, if not hedged appropriately. Therefore portfolio optimization with the objective of limiting financial exposure of companies has become a core concern for electricity companies around the world. Unfortunately so far in most liberalised electricity markets, hedging opportunities in the derivative markets remain limited. In sharp contrast to the optimistic expectations in the beginning of the liberalisation, the derivative markets for products with delivery beyond one or two years ahead are characterised by an astonishing lack of trading activity and thus liquidity. This implies that owners of physical generation assets will pay every hedging activity by reduced average earnings, given that the achievable sales price declines with increasing sales quantities. On the other hand, storage of the produced good is neither a hedging alternative for electricity producers, given that electricity is hardly storable at all. Thus portfolio optimization approaches are needed, which allow power producers to identify their optimal hedging strategies, taking into account the risk-return trade-off in the future markets and the risk preferences of the company respectively its shareholders.

Portfolio optimization has a long tradition in applied finance and in operations research. With the work of Markowitz (1952) , a standard approach has been made available, which allows portfolio selection taking into account risk and return aspects. The work of Sharpe (1964) and many others has made the role of risk in the portfolio selection for financial assets even more transparent and also the implication of portfolio selection on market equilibria in the 
finance markets has been established. In the (relatively) new field of electricity markets such a broad body of literature on portfolio selection does not exist. Obviously portfolio management is also a core aspect of liberalised electricity markets, yet the applications of standard portfolio optimization concepts remain limited. For the selection of power plant and fuel portfolios in the electricity industry, there have been various applications of mean-variance approaches starting with Bar-Lev and Katz (1976) and including also Humphreys and McClain (1998), Berger et al. (2003) and Krey and Zweifel (2006). Yet these applications do not analyse operational portfolio choices for hedging purposes yet the strategic issue, in which type of power plants, especially of which fuel type, to invest. Questions of operational portfolio management have been repeatedly dealt with using stochastic programming approaches. Work in this field includes Fleten et al.(2002), Eichhorn et al. (2005), Oum et al (2005), Weber (2005), Weber and Woll (2006). One broad strand of the literature is concerned with deriving optimal procurement strategies for utilities serving end users of electricity (in the U.S. often called "load serving entities"), such as the "Stadtwerke", the municipal utilities in Germany. These utilities have to face volume risk along with price risk and also have to deal with the fact that derivative markets are incomplete, i.e. hourly price risks can not be hedged by standard derivative products (e.g. Oum et al. (2005), Eichhorn et al. (2005)). Other approaches aim at identifying the optimal hedging strategies assuming liquid markets but production assets with multiple dynamic restrictions, such as water reservoirs Fleten et al. (2002) or CHP power plants Weber (2005), Weber and Woll (2006). The question of optimal portfolio selection with illiquid derivative markets has however hardly been addressed so far. In the context of portfolio optimization work on power plant systems at Aachen University, price-elastic bid functions have been integrated e. g. Hartmann et al. (2006), yet the portfolio optimization aspects in a mean-variance framework have not been addressed explicitly.

The present paper thus explicitly addresses this issue of optimal hedging strategy for power generators in a mean-variance framework. In the following section, the general model formulation is derived, taking the conventional Markowitz model as starting point. In order to 
arrive at a practically relevant approach, the model parameters have to be estimated carefully. This is done in the subsequent section, focusing notably on the variance-covariance matrix and the risk aversion parameters. The risk aversion parameter is thereby explicitly linked to the market price of risk as derived from CAPM models. In the application section subsequently the case of base electricity for delivery in 2008 on the German power exchange EEX is considered. It is notably shown, how the optimal hedging strategy depends on the size of the generation portfolio. The concluding section then provides final remarks and points at future research needs.

\section{Mean-Variance Model with limited liquidity}

Following Markowitz, a general mean-variance portfolio selection problem may be formulated as

$$
\max \left\{c^{\prime} x-\gamma x^{\prime} C x \mid A x \leq b\right\}
$$

where $c$ and $x$ are $\mathrm{n}$-dimensional vectors, $\mathrm{b}$ is a m-dimensional vector and $\mathrm{A}$ is a $n \times n$ matrix of constraints. $\mathrm{C}$ is a $n \times n$ matrix which is symmetric positive semidefinite and $\gamma$ is a scalar describing the risk aversion. We use here the notational convention that any vector $y$ is a column vector unless indicated to the contrary by transposition (e.g. $y^{\prime}$ ). The solution of (1) leads to the optimal Portfolio $x$ with respect to a chosen $\gamma$ under the given constraints. In traditional Mean-Variance models the objective is to maximize the risk-adjusted return of a portfolio of $n$ stocks. Here the returns are assumed to follow a multivariate normal distribution, i.e. $x \sim N(\mu, \Sigma)$. The corresponding problem formulation is

$$
\max \left\{\mu^{\prime} x-\gamma x^{\prime} \sum x \mid A x \leq b\right\}
$$

where $\mu$ is a n-dimensional vector of expected returns and $x$ a $n$-dimensional vector of portfolio weights. $\Sigma$ is a $n \times n$ positive definite covariance matrix and $\gamma$ the parameter for risk aversion again. In the context of stock portfolios, the only constraint is the budget constraint, i.e., $l^{\prime} x=1$, 
that dictates the portfolio weights to sum to one. Here $\imath$ denotes a vector of ones. So the model can be written as

$$
\max \left\{\mu^{\prime} x-\gamma x^{\prime} \sum x \mid \imath^{\prime} x=1\right\}
$$

The key difference of the portfolio selection model for power generation hedging is that now not different assets are considered but different sales dates for power to be produced e.g. in $2008^{1}$. This power to be produced in the future may be sold in advance on future or forward markets ${ }^{2}$, the most important derivative markets in the energy field. On these markets, firm contracts for power delivery in the future are negotiated, providing hence producers and consumers with a possibility to hedge themselves again highly volatile spot power prices. By entrying future or forward contracts, the producer can hence reduce his risk, but may face reduced prices due to limited market liquidity. Thus the vector $x=\left(x_{1}, \ldots, x_{t}, \ldots, x_{T}\right)^{\prime}$ describes in this case the sales quantities in different contracting periods, e.g. all the months from today until power delivery in 2008. The objective is then to optimize the risk-adjusted expected value of the receipts $p x$, with the risk being again measured through the variance of the receipts:

$$
\max \left\{\mathrm{E}[p x]-\gamma \operatorname{Var}[p x] \mid l^{\prime} x=V_{0}\right\},
$$

$V_{0}$ is thereby the total quantity to be sold, $\gamma$ describes again the degree of risk aversion, $p=\left(p_{1}, \ldots, p_{T}\right)^{\prime}$ is a given price vector and I the identity matrix.

Most straightforwardly, liquidity limitations may be modelled through a linear pricedemand function ${ }^{3}$ for each period $t$ :

$$
p_{t}=p_{o, t}-\alpha_{t} x_{t}
$$

\footnotetext{
1 The basic issues are best dealt with in a context of just one delivery period. Obviously the problem may be generalized to cover several delivery periods, such as the years 2008 to 2010 .

2 The main difference between future and forward contracts is that the former are traded on power exchanges whereas the latter are traded bilaterally in the so-called OTC-market. Moreover for future contracts in general cash settlement is foreseen, whereas forwards mostly include the possibility of physical delivery (cf. e.g. Hull (2006)). However none of these differences are relevant in the problem formulation treated here.

3 Obviously also other forms of a price-demand function may be stipulated, yet the linear specification has the clear advantage that the overall problem remains a quadratic optimisation problem, as shown below.
} 
The parameter $p_{o, t}$ herein describes the "undisturbed" price at sales quantity 0 , the (time-dependent) parameter $\alpha_{t}$ the slope of the price-demand function. The higher the liquidity is in the market, the lower the value of $\alpha_{t}$. The undisturbed price $p_{o, t}$ is taken as a normally distributed random variable, whereas for $\alpha_{t}$ no stochasticity is assumed, in order to keep the problem quadratic. The distribution of $p_{o}=\left(p_{o, 1}, \ldots, p_{o, T}\right)^{\prime}$ is taken as multivariate normal, with $p_{o} \sim N\left(p_{0} l, C\right)$. The expected undisturbed price for all future periods is set equal to $p_{0}$, because otherwise there would be systematic incentives for arbitrage trading. I.e. the price process for $p_{o, t}$ is assumed to fulfil the so-called martingale property, i.e. $\mathrm{E}\left[p_{o, t}\right]=p_{0}$.

Using the definition of the variance and the fact that neither $\alpha=\left(\alpha_{1}, \ldots, \alpha_{T}\right)^{\prime}$ nor $x_{t}$ are stochastic, it can be shown that the problem (4) is equivalent to:

$$
\max \left\{\left(p_{0} \imath-\alpha I x\right)^{\prime} x-\gamma x^{\prime} C x \mid \imath^{\prime} x=V_{0}\right\}
$$

This quadratic optimization problem with (one) equality constraint may be solved using the Lagrangian

$$
L(x, \lambda)=\left(p_{0} l-\alpha I x\right)^{\prime} x-\gamma x^{\prime} C x-\lambda\left(\imath^{\prime} x-V_{0}\right)
$$

The first-order conditions for an optimum are then:

$$
\begin{aligned}
& \frac{\partial L}{\partial x}=p_{0} l-2 \alpha I x-2 \gamma C x-\lambda l=0 \\
& \frac{\partial L}{\partial \lambda}=\iota^{\prime} x-V_{0}=0
\end{aligned}
$$

These form a linear equation system of full rank, if $C$ is positive semi-definite:

$$
A w=b
$$

with

$$
A=\left[\begin{array}{cc}
-2 \alpha I-2 \gamma C & \imath \\
\iota^{\prime} & 0
\end{array}\right] \quad w=\left[\begin{array}{c}
x \\
\lambda
\end{array}\right] \quad b=\left[\begin{array}{c}
-p_{0} \imath \\
V_{0}
\end{array}\right]
$$

Thus a unique solution vector $x$ of optimal sales quantities together with a shadow value $\lambda$ for each additional unit of $V_{0}$ may be determined by solving the equation system (9). It should be noted that the quantities $x$ are optimal from the view point of today. The decision problem however has a wait-and-see structure (cf. Birge and Louveaux (1997)), i.e. only the first decision 
$x_{1}$ has to be taken now. The others may be postponed and consequently may be modified later, if in the mean time new information has arrived.

\section{Model Calibration}

For the practical application of the model, the appropriate choice of the parameters is crucial. Given the martingale property of the future prices, the price $p_{0}$ should be set equal to the last observed price. Also the quantity $V_{0}$ is easily determined, since it has to be chosen by the user. More difficult are by contrast the choices of $\alpha, \gamma$ and $C$.

The slope parameter $\alpha$ of the price-sales function depends on the liquidity of the wholesale market. Thereby various interlinked difficulties arise. Firstly, the liquidity of a market is not directly observable. Rather different concepts are typically used for measuring approximately the liquidity Kempf (1999). For example, sometimes the traded volumes are taken as indicators for the liquidity in the market. However these do not provide a precise indication on the prices, at which sales may be done. Secondly, the slopes of the market supply or demand functions are mostly not directly observable. Power exchanges, like other exchanges, often publish best bid and best ask prices $p_{B}$ and $p_{A}$. Yet which quantities are traded or could be traded at this price is mostly not known - at least to non-market participants. Here $\alpha$ is therefore estimated based on the observable bid-ask spread. With the reservation price $p_{R}$ being taken as the average of the bid and ask prices, the absolute value of the slope is obtained from:

$$
\alpha=\frac{p_{R}-p_{B}}{q}=\frac{\frac{p_{B}+p_{A}}{2}-p_{B}}{q}=\frac{p_{A}-p_{B}}{2 q}
$$

Based on average values for the base product year 2008, a value of $0,0035 € / M W$ is obtained for

$\alpha$

The covariance matrix $C$ may be derived from historical observations or from theoretical considerations or from a combination of both. The most sophisticated way of estimating $C$ is to use a multifactor model of commodity prices, such as the ones investigated by Gibson and Schwartz (1990), Schwartz (1997), Clewlow and Strickland (2000) or Lucia and Schwartz 
(2002). However, the outcomes of these models are rather sensitive to specification choices and also these models are often beyond the reach of practitioners. Therefore in the following simpler approaches are applied to identify appropriate covariances.

The first approach is to assume a random walk pattern for the future prices with independently identically distributed (i.i.d.) steps. The variance of price changes over $t$ periods can then be obtained from (cf. e.g. Hull (2006)):

$$
\sigma_{t}^{2}=t \sigma_{1}^{2}
$$

The covariance between price changes over $i$ and $j$ periods is given through

$$
{\sigma_{i, j}}^{2}=\min (i, j) \sigma_{1}^{2}
$$

since changes over two periods are identical for the overlapping part of the two periods (i.e. the minimum of $i$ and $j$ ) and independent for the remainder.

Hence the first covariance matrix $C_{1}$ to be used is given by:

$$
C_{1}=\left(\begin{array}{ccccc}
0 & 0 & 0 & \cdots & 0 \\
0 & \sigma_{1}{ }^{2} & \sigma_{1}{ }^{2} & \cdots & \sigma_{1}{ }^{2} \\
0 & \sigma_{1}^{2} & 2 \sigma_{1}^{2} & \cdots & 2 \sigma_{1}{ }^{2} \\
\vdots & \vdots & \vdots & \ddots & \vdots \\
0 & \sigma_{1}^{2} & 2 \sigma_{1}^{2} & \cdots & t \sigma_{1}^{2}
\end{array}\right)
$$

Thereby it is assumed that the first possible action is immediate trading without stochastic price risk. The next trading opportunity is after one period (e.g. one month) and so on.

This approach makes rather strong theoretical assumptions and uses empirical evidence only to determine the standard deviation $\sigma_{1}$. Alternatively, if enough observations are available, one may compute all the elements of the covariance matrix from empirically observed time series. This requires the calculation of time series differences over several lag periods:

$$
d p_{t, i}=p_{t+i}-p_{t}
$$

The matrix $C_{2}$ is then obtained by computing the covariances on all pairs $d p_{t, i}$ and $d p_{t, j}$ :

$$
C_{2}=\left\{\operatorname{Cov}\left(d p_{t, i}, d p_{t, j}\right)\right\}
$$


When computing these covariances, attention has to be paid to take the time series $d p_{t, i}$ always over the same observation period. Otherwise the obtained covariance matrix may not be positive semidefinite.

But even if this is the case, spurious correlations in the data may lead to a covariance structure, which imply exploitable market inefficiencies. E.g. the introduction of the $\mathrm{CO}_{2}$ trading scheme has lead to a continuous upwards price trend in the German data in the period march 2005 to march 2006, whereas there was not such an obvious trend before 2005. Therefore a (small) positive correlation of price changes between subsequent months appears in the data - whereas it can not be expected to persist in the future. Thus the data should be corrected for such spurious effects. This can be done by maintaining the diagonal elements of the empirical covariance matrix but correcting the off-diagonal elements using the same rule as for matrix $C_{1}$. Thus the elements $c_{3, i j}$ of the covariance matrix $C_{3}$ are given by:

$$
c_{3, i j}=\operatorname{Var}\left(d p_{t, \min (i, j)-1}\right)
$$

The risk aversion coefficient $\gamma$ can be set by the model user according to some expressed preferences of the management. Yet the quantity is difficult to explain and difficult to provide a reasonable estimate for. Moreover for a market-oriented decision making, the value retained should be preferably linked to some market characteristics. One such market characteristic is the market price of risk, as derived from the CAPM model Sharpe (1964). Risk in the electricity market should be sold off by the power generator, if the price for the risk reduction is lower than the market price of corresponding risk. The market price of risk is expressed by the socalled Sharpe ratio $R_{\text {Sharpe, }}$ defined through:

$$
R_{\text {Sharpe }}=\frac{r_{M}-r_{0}}{\sigma_{M}}
$$

Thereby $r_{M}$ is the average return of the market portfolio in the stock market, and $\sigma_{M}$ the corresponding standard deviation. A long-term average for the Sharpe ratio is about 0.3 (see, e.g. Dimson et al. (2006)), although the estimates vary considerably, depending on the methods applied and the observation period considered. The return on electricity price risk taken, i. e. 
the increase in expected receipts, should then in the optimum be equal to the Sharpe ratio multiplied by the systematic part of the electricity price risk, i.e. the part which is correlated with the general market risk. This part is difficult to evaluate, given that the current market is still in transition. Yet a proxy is to take the so-called betas of stock-market listed utilities with heavy generation assets, e.g. RWE and E.ON. Whereas the beta used to be between 0.5 and 0.7 , it has risen to 1 in the year 2006.

$$
\frac{d(\mathrm{E}[p x])}{d \gamma}=R_{\text {Sharpe }} \tilde{\beta} \frac{d(\sigma[p x])}{d \gamma}
$$

Determining the return on risk $(R o R)$ analytically is hardly possible. Yet through a variation of the risk parameter $\gamma$, the risk-return trade-off can be determined for efficient portfolios and plotted graphically, as in the $\mu$ - $\sigma$-diagram of the standard Markowitz model (cf. Figure 1).

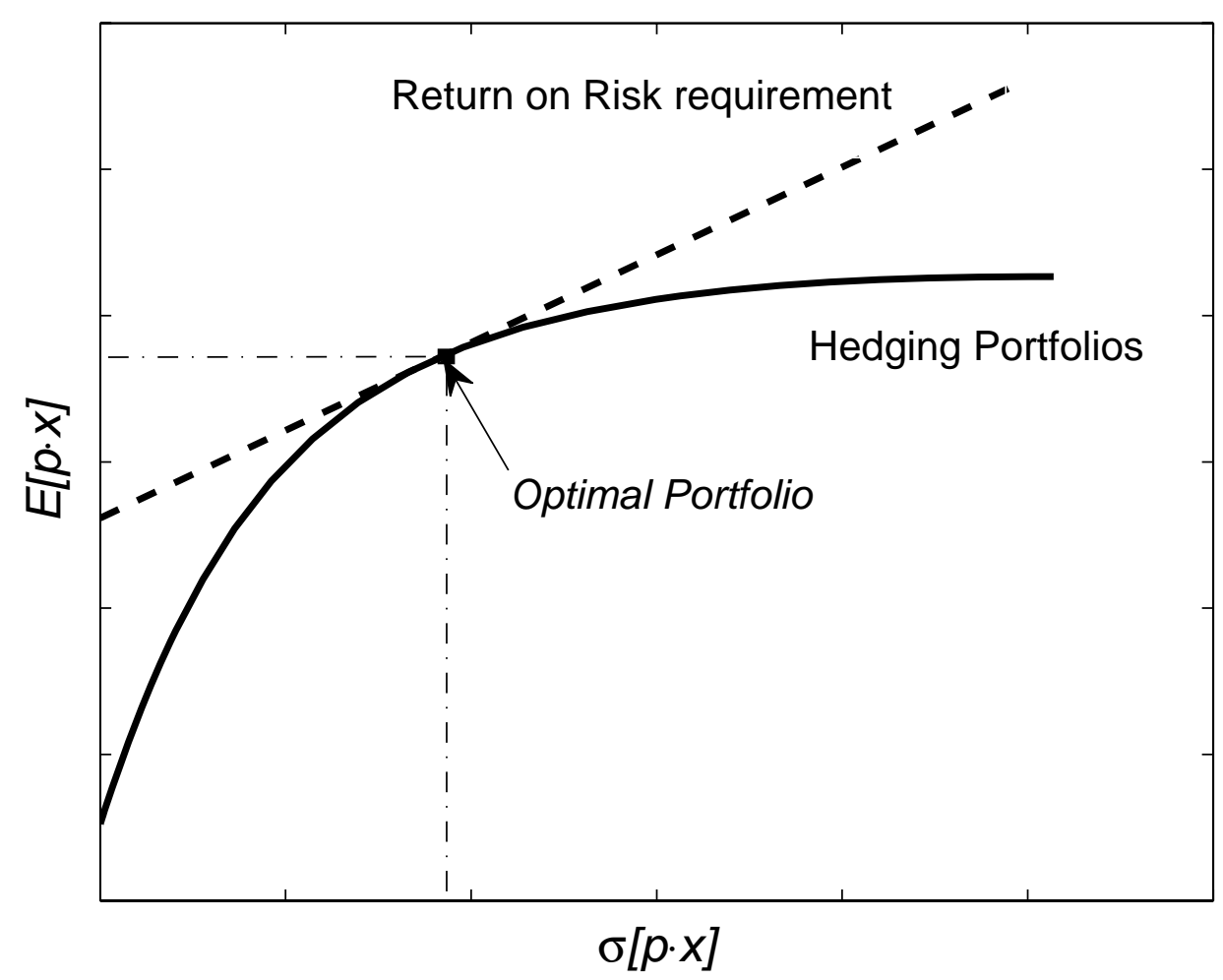

Figure 1: $\quad$ Risk-return trade-off for efficient electricity hedging portfolios 
For determining the optimal portfolio numerically, a direct computation of the return on risk using numerical differences is however preferable (cf. Figure 2).

$$
\operatorname{RoR}=\frac{d(\mathrm{E}[p x])}{d(\sigma[p x])}=\frac{\Delta_{\gamma}\left(\left(p_{0} l-\alpha I x^{*}\right)^{\prime} x^{*}\right)}{\Delta_{\gamma}\left(x^{* *^{\prime}} C x^{*}\right)}
$$

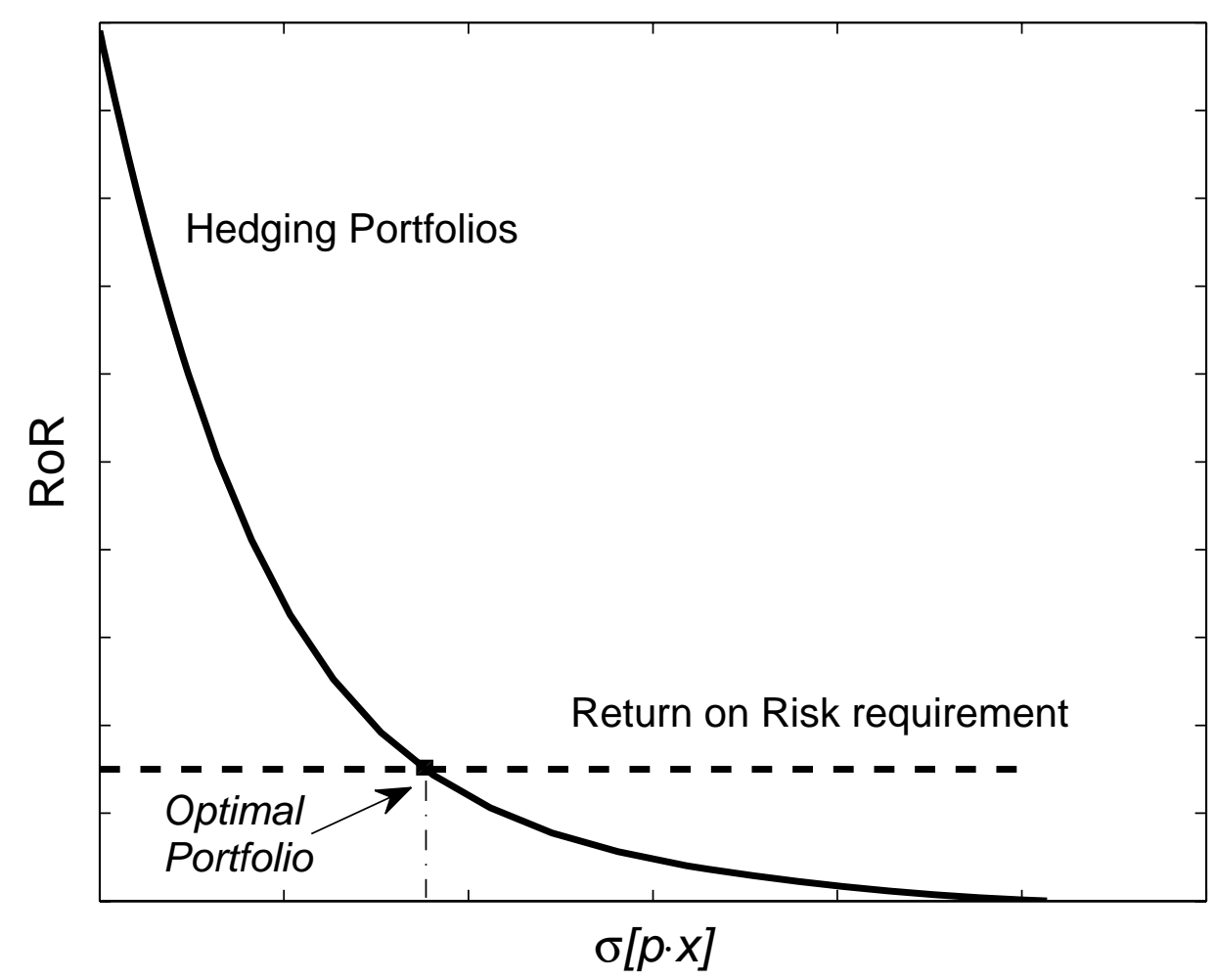

Figure 2: $\quad$ Return on risk for efficient electricity hedging portfolios

\section{Application}

The developed approach is applied exemplarily for the hedging of a base electricity production, i.e. a continuous band delivery for the year 2008. Placing ourselves at the end of 2006, we aim at identifying the optimal sell-off strategy for this product in the derivative markets. The price level at the end of 2006 was $56 € / M W h$. For $1000 \mathrm{MW}$, i.e. the power of a typical large-scale power plant, delivered $8760 \mathrm{~h}$ in a year, this means total receipts of 490 Mio. $€$, if no rebates have to be given. 
The corresponding risk-return profile of efficient portfolios is depicted in Figure 3, taking the covariance matrix $C_{1}$ as basis. Obviously the shape of the profile is not a parable, as in the traditional Markowitz model. Rather there is a flat maximum achievable for risk neutral decision makers (upper right end of the curve). This maximum corresponds to a strategy of continuous sales at a constant rate, because in this case the reduction of the sales price is lowest on average. At the opposite left end of the curve, a cash flow of 461 Mio. $€$ may be obtained, if the whole electricity is sold off at once. Then no risk is incurred. In between the return on risk is continuously decreasing, i.e. the function is concave. Correspondingly, the first order derivative depicted in Figure 4 is monotonously falling. The required return-onrisk level is reached with a standard deviation of 20,8 Mio. €, the corresponding expected receipts are 487,7 Mio. €, and the parameter $\gamma$ takes the value 0.0001 .

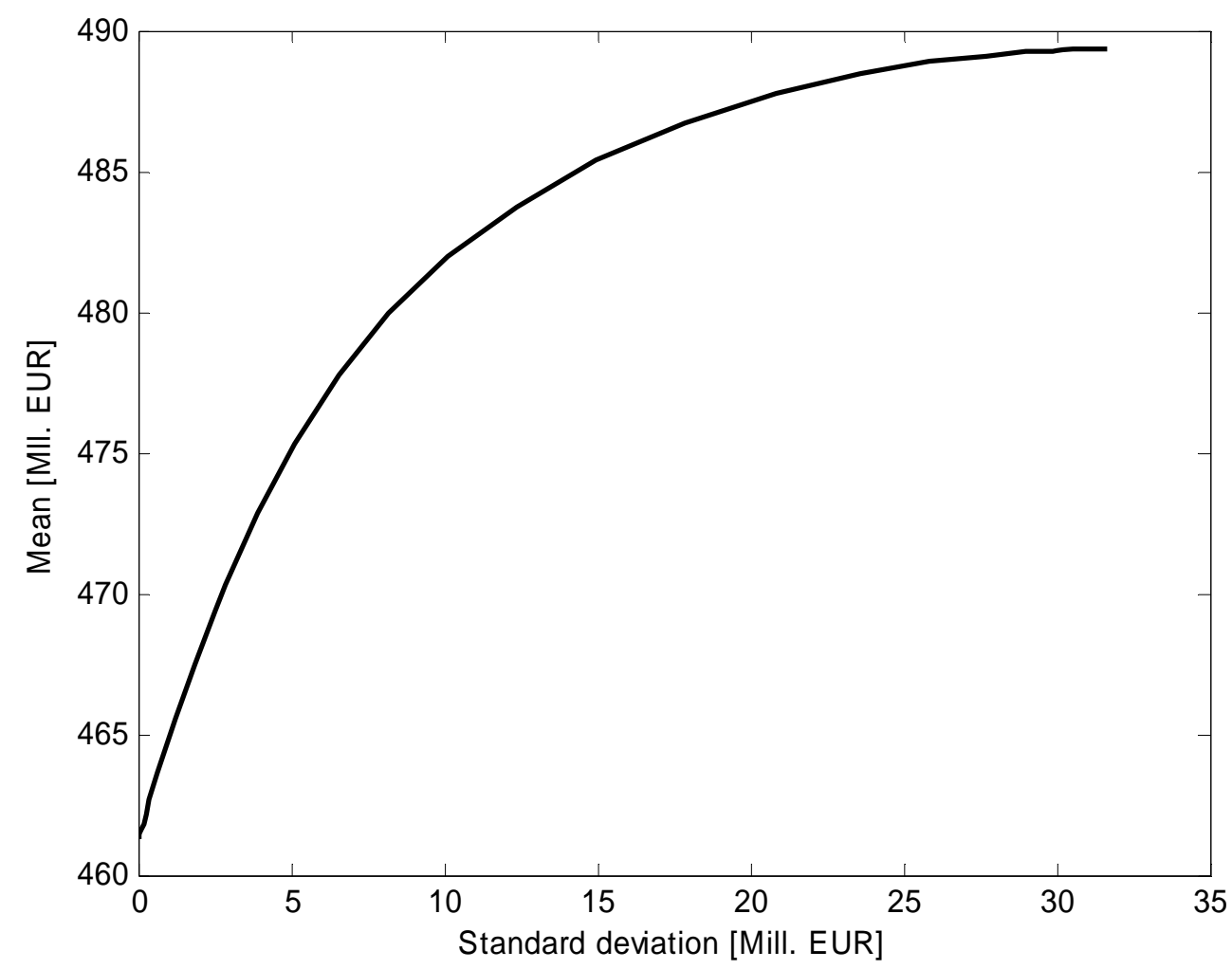

Figure 3:Risk-return trade-off for efficient electricity hedging portfolios, reference application case 


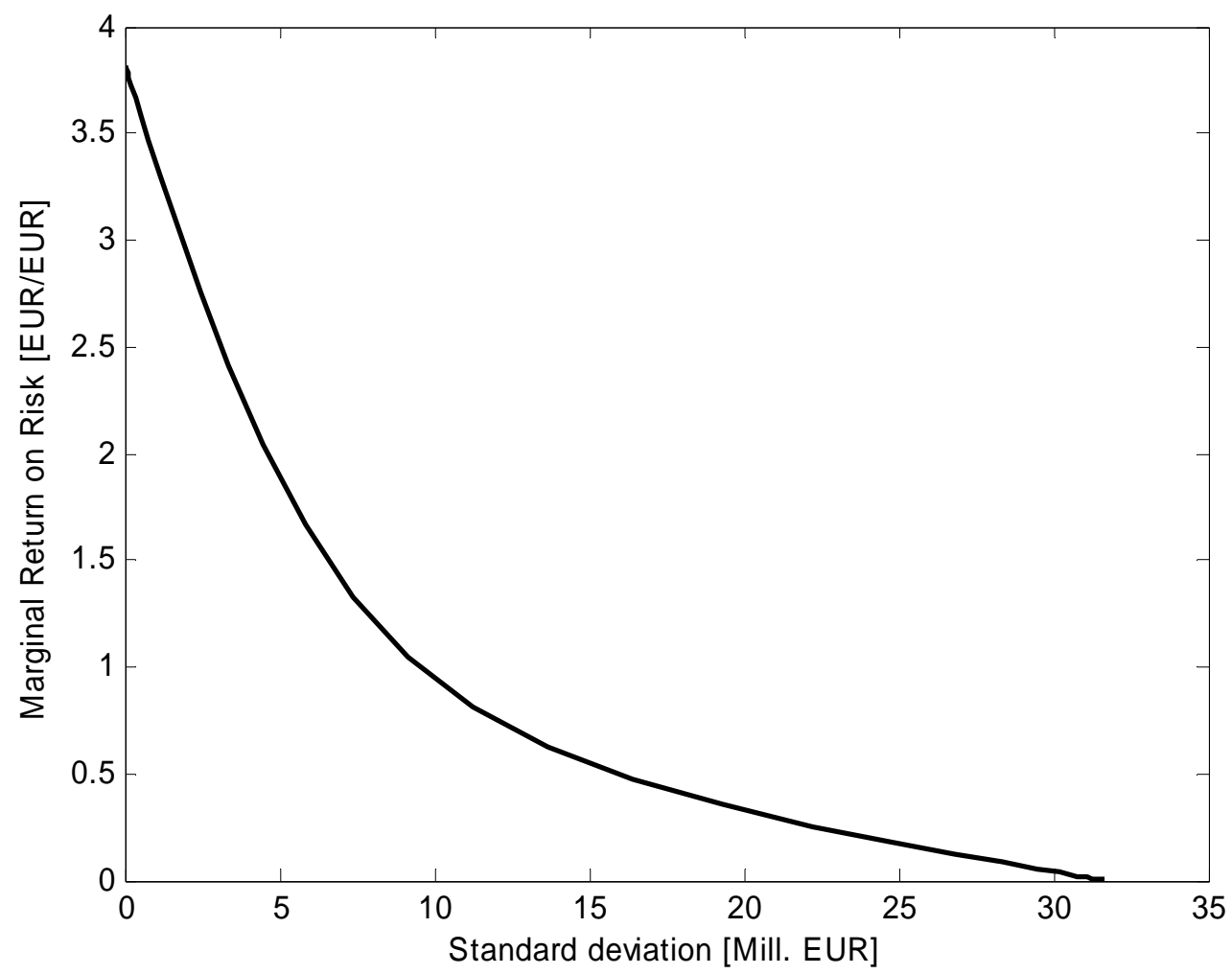

Figure 4:Return on risk for efficient electricity hedging portfolios, reference application case

The optimal hedging strategy is depicted in Figure 5. Obviously in this case it is preferable to sell off more in the beginning, where the price risks are limited. But still nearly half of the total power is kept for the months 4 to 12 , in order to avoid negative effects on sales prices. 


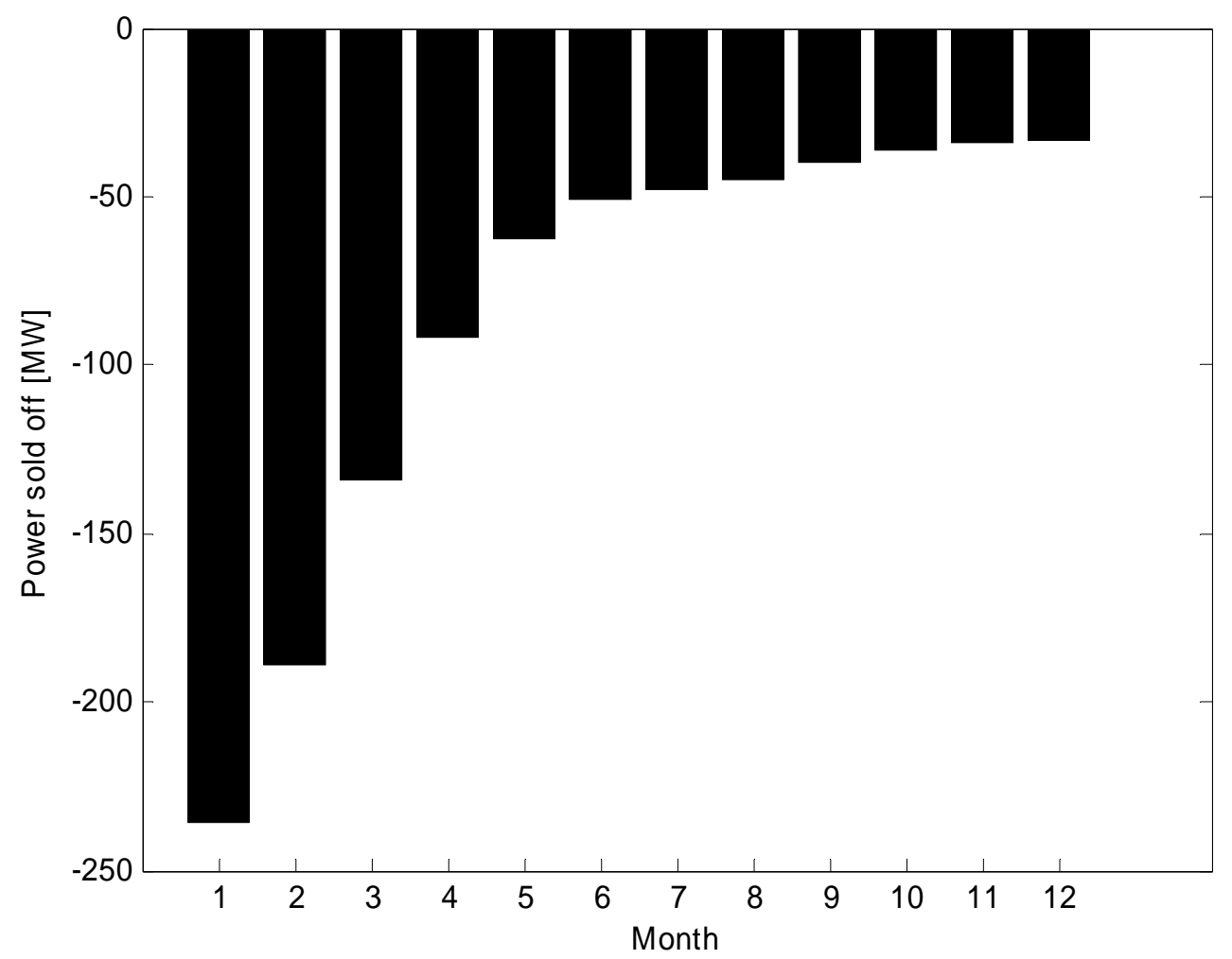

Figure 5: $\quad$ Optimal hedging strategy, reference application case

The impact of the assumed covariance structure on the results becomes apparent when looking at the following pictures. Figure 6 illustrates that the achievable expected returns in the no-risk case and in the risk-neutral case are similar for all covariance matrices. But the shape of the risk-return profile is different. And in some cases the optimal hedging strategy involves overselling when using the empirical covariance matrix. This can occur if positive correlations are included in this matrix, which make it profitable to anticipate future price changes and to buy back later on presumably better prices. Since such expectations are rather speculative ex-ante, the necessity for correcting the empirical covariance matrix is evident. The corrected version then indicates that sales should optimally be postponed somewhat longer than suggested by the Brownian motion approach. The reason is that price variance over a longer period tends to increase less than linearly. Consequently the risk exposure through open positions is less than assumed in the simple model. 


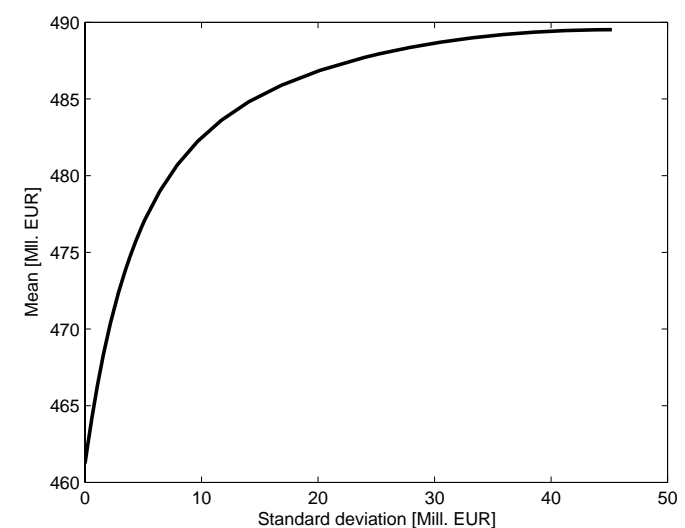

a) empirical covariance matrix

Figure 6:Risk-return trade-off for efficient covariance matrices

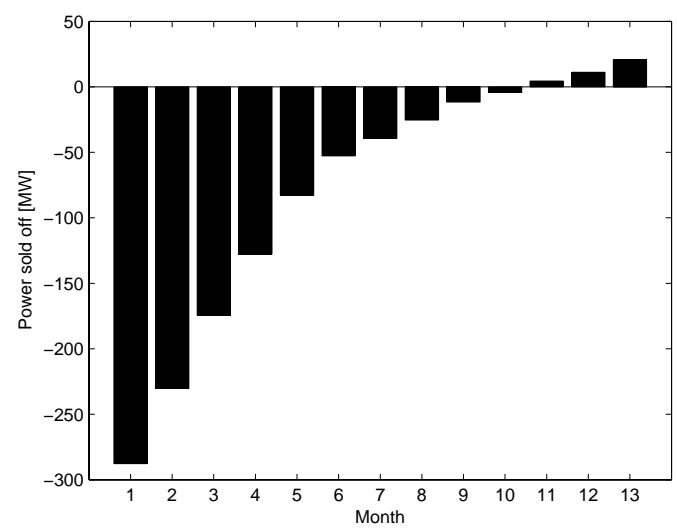

a) empirical covariance matrix

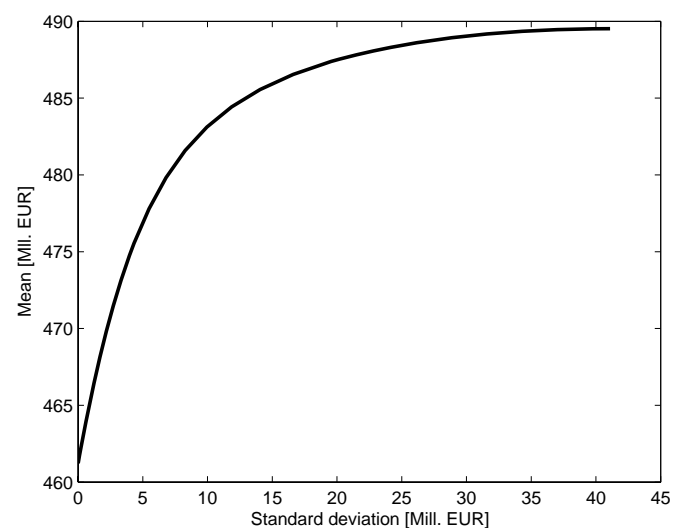

b) empirically based but theoretically consistent covariance matrix

electricity hedging portfolios, alternative

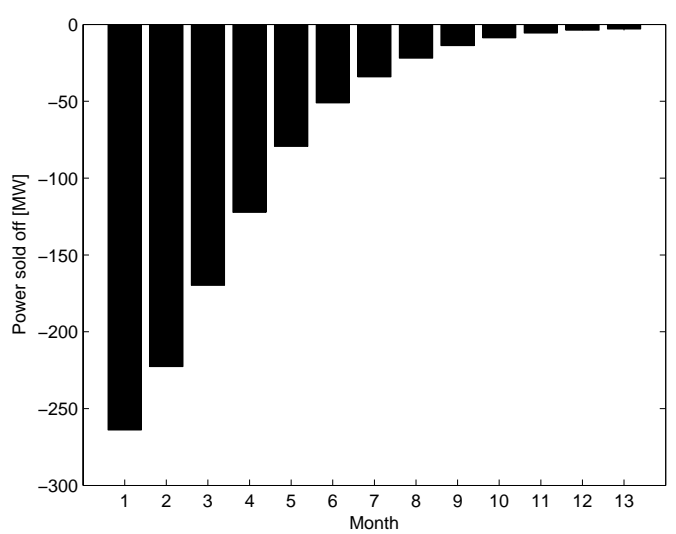

\section{Figure 7: $\quad$ Optimal hedging strategy, alternative covariance matrices}

Contrarily to the classical Markowitz model, the hedging portfolio optimization model is not scale invariant. When reducing the power to be sold from $1000 \mathrm{MW}$ to $300 \mathrm{MW}$, the optimal hedging strategy shifts towards earlier hedging (cf. Figure 9). The reason becomes obvious when looking at the risk-return profile in Figure 8. The smaller quantities lead to lower price decreases. So instead of loosing more than $6 \%$ of the portfolio value when selling off immediately, the losses are restricted to about $0.8 \%$ in the case of the smaller generation power. Then of course the return on risk gets accordingly smaller, and it is preferable to hedge earlier. In the conventional Markowitz model, the price dumping effect does not exist. Therefore the optimal shares in the portfolio are independent of the size of the portfolio. 


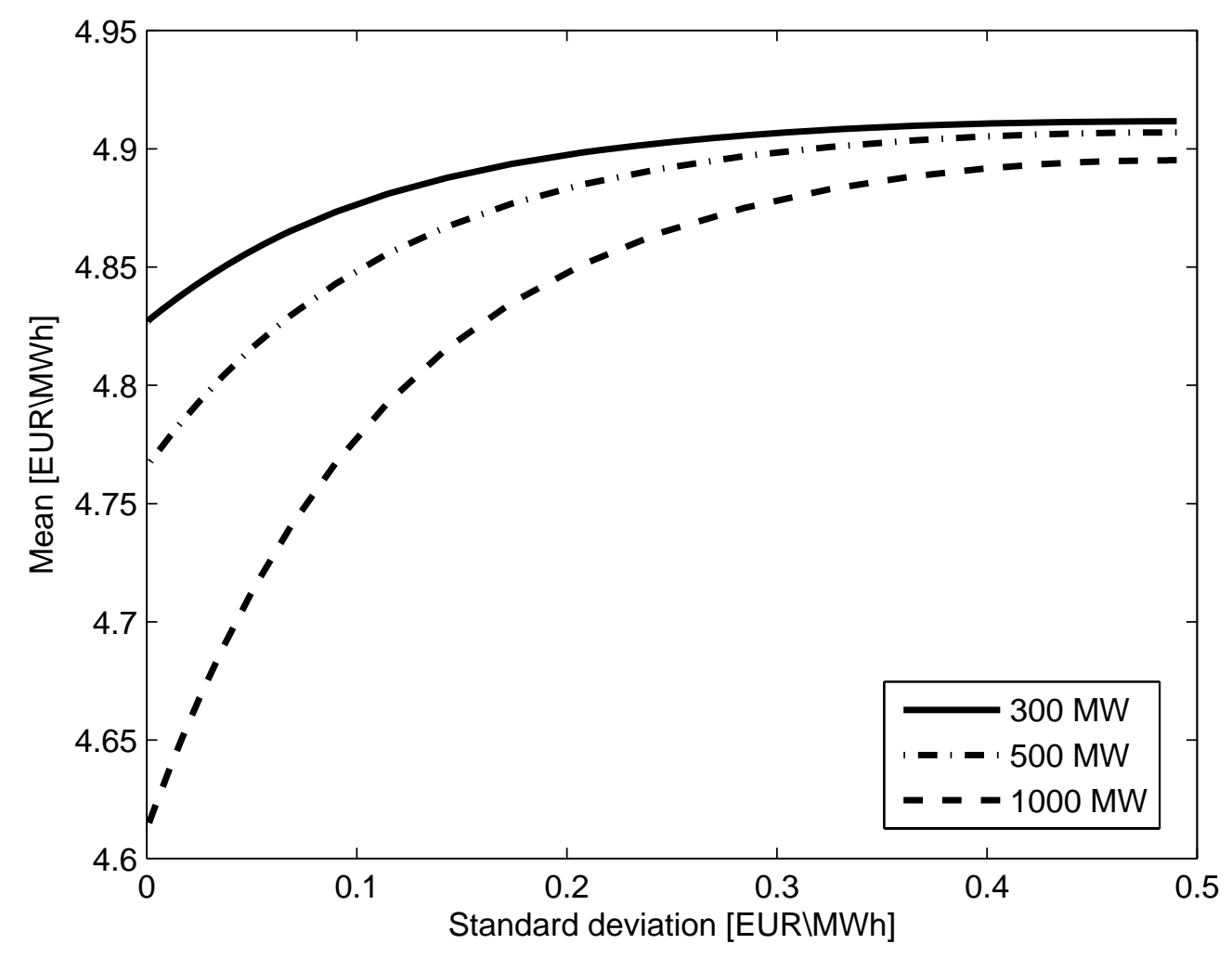

Figure 8: $\quad$ Risk-return trade-off for efficient electricity hedging portfolios

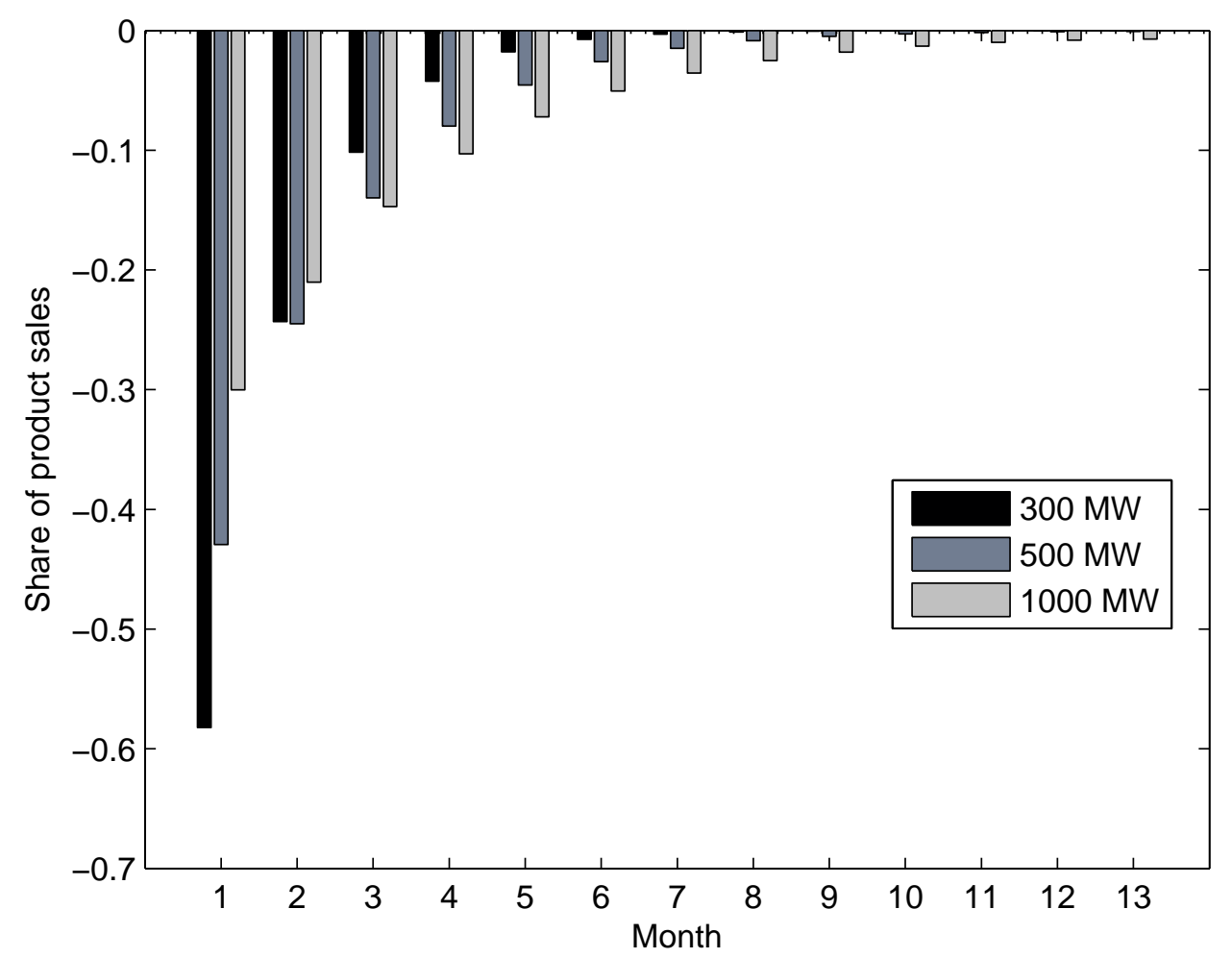

Figure 9:

Optimal hedging strategies 


\section{Summary}

A mean-variance approach for hedging portfolio optimization has turned out to be a valuable tool for portfolio management in the electricity industry. At first sight the approach looks rather similar to conventional optimization approaches for stock portfolios, yet the outcomes clearly illustrate that devising an optimal hedging strategy in future markets with limited liquidity is different from optimising the risk-return trade-off in conventional asset portfolios. One key difference is that the size of the portfolio matters. Hedging is particularly a challenge when it comes to portfolios of some $300 \mathrm{MW}$ or even some $1000 \mathrm{MW}$.

Obviously the approach presented here might be developed further for encompassing further products, both in electricity and in the related fuel and $\mathrm{CO}_{2}$ markets. Also the time-dependency of liquidity requires a more detailed investigation. Finally also the use of more advanced price models for future prices may be worth looking at.

\section{References}

Bar-Lev, D., S. Katz. 1976. A Portfolio Approach to Fossil Fuel Procurement in the Electric Utility Industry. Journal of Finance June 31 (3) 933-947.

Berger, M., S. Awerbuch, R. Haas. 2003. Versorgungssicherheit und Diversifizierung der Energieversorgung in der EU. Bundesamt für Verkehr, Innovation und Technologie, Wien: Berichte aus Energie- und Umweltforschung 2 .

Birge, J.R., F. Louveaux. 1997. Introduction to stochastic Programming. Springer, New York .

Clewlow, L., C. Strickland. 2000. Energy derivatives. Pricing and risk management. Lacima Publications, London.

Dimson,E., P. Marsh, M. Staunton. 2006. The Worldewide Equity Premium: A smaller puzzle. EFA 2006 Zürich Meetings Paper, Zürich.

Eichhorn, A., W. Römisch,I. Wegener. 2005. Polyhedral risk measures and lagrangian relaxation in electricity portfolio optimization. Dagstuhl Seminar Proceedings 05031-Algorithms for Optimization with Incomplete Information, Dagstuhl. 
Fleten, S.-E., S. W. Wallace, W. T. Ziemba. 2002. Hedging Electricity Portfolios Via Stochastic Programming. IMA Volumes on Mathematics and Its Applications 128 71-93.

Gibson, R., E. S. Schwartz. 1990. Stochastic Convenience Yield and the Pricing of Oil Contingent Claims. The Journal of Finance 45959 - 976.

Hartmann, T., B. Blaesig,, G. Hinüber,H.-J. Haubrich. 2006. Stochastic Optimization in Generation and Trading Planning. Operations Research Proceedings 2006.

Hull, J. 2006. Options, futures and other derivates. Pearson Studium, München..

Humphreys, H., K. McClain. 1998. Reducing the Impacts of Energy Price Volatility through Dynamic Portfolio Selection. The Energy Journal 19 (3) 107-131.

Kempf, A. 1999. Wertpapierliquidität und Wertpapierpreise. Beiträge zur betriebswirtschaftlichen Forschung 91, Gabler Verlag, Wiesbaden.

Krey, P., P. Zweifel. 2006. Efficient Electricity Portfolios for Switzerland and the United States. http//www.soi.unizh.ch Working Paper No. 0602, retrieved 2006-11-22.

Lucia, J. J.,E. S. Schwartz. Electricity prices and power derivatives: Evidence from the Nordic power market. Review of Derivative Research 55 - 50.

Markowitz, H. 1952. Portfolio Selection. Journal of Finance 7 77-91.

Oum, Y., S. Oren,S. Deng, 2005. Hedging Quantity Risks with Standard Power Options in a Competitive Wholesale Electricity Market. Naval Research Logistics 53 (7) 697-712.

Schwartz, E. S. 1997. The Stochastic Behavior of Commodity Prices: Implications for Valuation and Hedging. The Journal of Finance 52923 - 973.

Sen, S., L. Yu, T. Genc. 2002. A Stochastic Programming Approach to Power Portfolio Optimization. Institute for Operations Research and the Management Sciences : Operations research 54 (1) 55-72.

Sharpe, W. 1964. Capital Asset Prices: A theory of Market Equilibrium under Conditions of Risk. Journal of Finance 19425 - 442.

Unger, G. 2006.: Hedging Strategy and Electricity Contract Engineering.http://ecollection.ethbib.ethz.ch/ecol-pool/diss/fulltext/eth14727.pdf, retrieved 2006-11-10. 
Vehviläinen, I., J. Keppo. 2003. Managing electricity market price risk. European Journal of Operational Research 145136 - 147.

Weber, C. 2005. Uncertainty in the Electric Power Industry. Methods and Models for Decision Support. Springer, New York 2005.

Weber, C., O. Woll. 2006. Portfolio Optimization for CHP portfolios. Proceedings PMAPS Stockholm 2006.

Yu, Z. 2003 A Spatial Mean-Variance MIP Model for Energy Market Risk Analysis. Energy Economics 25 255-268. 